
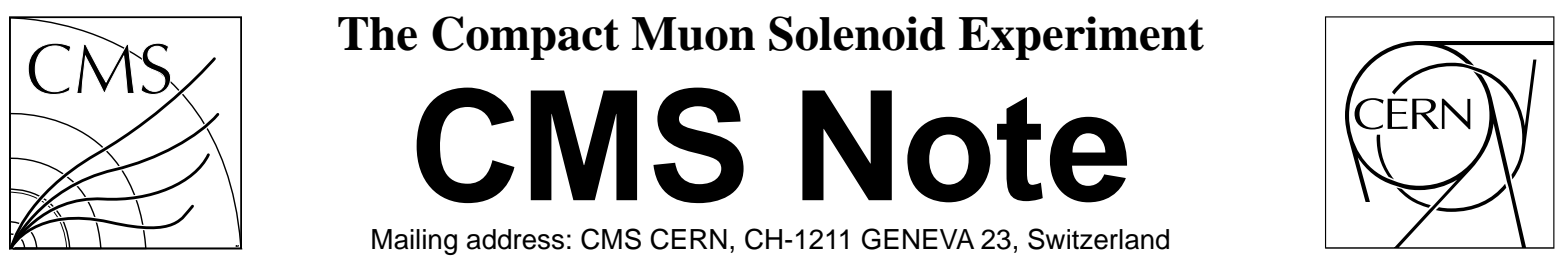

6 June 2006

\title{
Observability of bbZ events at CMS as a benchmark for MSSM bbH search
}

\author{
S. Lehti
}

Helsinki Institute of Physics, Helsinki, Finland

\begin{abstract}

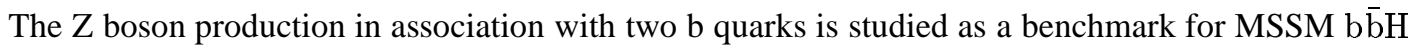
production with $\mathrm{H} \rightarrow \tau \tau$ decay in CMS. An analysis using $\mathrm{Z}$ bosons in real data can be used to test the methods and techniques needed for reconstructing the Higgs boson. The $b \bar{b} Z$ measured from data can be used to verify the theoretical predictions for the cross section, and $\mathrm{Z}$ boson and $\mathrm{b}$ quark transverse momentum distributions. Understanding the $\mathrm{b} \overline{\mathrm{b} Z}$ production at $\mathrm{LHC}$ helps us to understand and better trust the predictions for the associated Higgs bosons production.
\end{abstract}




\section{INTRODUCTION}

In MSSM the associated Higgs boson production gg/qq $\rightarrow \mathrm{b} \overline{\mathrm{b}} \mathrm{H} / \mathrm{A}$ with decay to tau pairs $\mathrm{H} / \mathrm{A} \rightarrow \tau \tau$ is important at large values of $\tan \beta$ due to enhanced Higgs boson couplings to down type fermions. This makes the H/A $\rightarrow \tau \tau$ a potential discovery channel for heavy neutral Higgs bosons $\mathrm{H}$ and $\mathrm{A}$. The $\mathrm{Z}$ boson production process with associated $\mathrm{b}$ jets $\mathrm{gg} / \mathrm{qq} \rightarrow \mathrm{b} \overline{\mathrm{bZ}}$ is topologically similar to the Higgs production process $\mathrm{gg} / \mathrm{qq} \rightarrow \mathrm{b} \overline{\mathrm{b} H} / \mathrm{A}$ as shown in Fig. 1. This similarity can be exploited by studying the gg/qq $\rightarrow \mathrm{b} \overline{\mathrm{b} Z}$ production using real data [1]. This allows one to compare the measured and predicted $\mathrm{b} \overline{\mathrm{b} Z}$ cross section, distributions for the $\mathrm{Z}$ boson transverse momentum, and the transverse momenta and pseudorapidity distributions of the associated $b$ jets to verify NLO calculations and kinematics. If the theoretical predictions for $\mathrm{Z}$ bosons are verified with real data, the predictions for the associated Higgs boson production should apply, too.

This study concentrates on $\mathrm{Z}$ bosons decaying in fully leptonic final states. The $\mathrm{Z}$ boson is also allowed to decay to leptons via intermediate taus. The leptonic final state gives a clean signal which is easy to trigger. The $\mathrm{Z}$ boson transverse momentum measurement is also accurate.

The $\mathrm{b} \overline{\mathrm{bZ}}$ production at LHC can also be used as a benchmark for $\mathrm{H} / \mathrm{A} \rightarrow \tau \tau$ [2]. The $\mathrm{Z}$ mass is known with an excellent precision, which can be used to verify the mass reconstruction method. Unlike the Higgs boson with couplings proportional to lepton mass, the direct decay of the $\mathrm{Z}$ boson to electron or muon pairs is not suppressed compared to tau. In testing the mass reconstruction method it is important that events with intermediate taus are selected. This can be achieved by choosing e $\mu$ final states only.
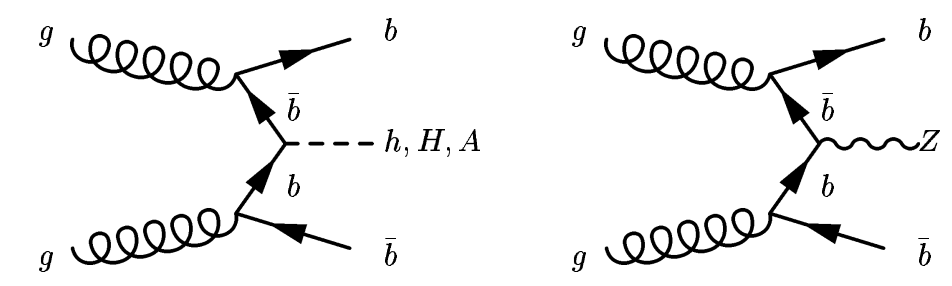

Figure 1: Example LO Feynman graphs contributing to Higgs and $\mathrm{Z}$ boson production in association with $\mathrm{b}$ quarks.

\section{SIMULATION OF SIGNAL AND BACKGROUND}

The signal consists of $\mathrm{Z} / \gamma^{*}$ events produced in association with $\mathrm{b}$ quarks emitted mostly in the forward direction. The $\mathrm{Z}$ boson and $\gamma^{*}$ are allowed to decay to electron, muon or tau pairs, tau decaying leptonically. The signal $\left(\mathrm{b} \overline{\mathrm{bZ}} / \gamma^{*}\right)$ is generated, and the corresponding cross section is calculated with CompHEP [3]. The resulting LO signal cross section for any-two-lepton final state is $58 \mathrm{pb}$. No $\mathrm{p}_{\mathrm{T}}$ or $\eta$ cuts were applied on b quarks in the $\mathrm{b} \overline{\mathrm{b} Z} / \gamma^{*}$ process generation. Nonzero mass is used for $\mathrm{b}$ quarks throughout all the calculation.

The background comes mainly from two sources, $\mathrm{Z} / \gamma^{*}$ associated with light quark and gluon jets, generated with PYTHIA [4], and t $\bar{t}, \mathrm{tW}$ events, generated with TopREX [5]. An NLO cross section of $1891 \mathrm{pb}$ calculated with MCFM [6] is used for Drell-Yan $\mathrm{Z} / \gamma^{*} \rightarrow \ell \ell(\ell=\mathrm{e}, \mu$ or $\tau)$ events with $\mathrm{m}_{\ell \ell}>80 \mathrm{GeV} / \mathrm{c}^{2}$. For $\mathrm{t} \overline{\mathrm{t}}$ and $\mathrm{tW}$ a cross section of, respectively, 840 and $60 \mathrm{pb}$ is used [7]. The $\mathrm{Z} / \gamma^{*}$ background sample consists also of events with two associated $\mathrm{b}$ quarks, but to prevent double counting, those events are removed using the available generation level information of the events.

As shown in Table 1, the cross section for $\mathrm{gg} / \mathrm{qq} \rightarrow \mathrm{b} \overline{\mathrm{bZ}} / \gamma^{*}, \mathrm{Z} / \gamma^{*} \rightarrow \tau \tau$ in fully leptonic final states is quite small, in fact of the order of $\mathrm{b} \overline{\mathrm{b}} \mathrm{H} / \mathrm{A}$ cross section at $\mathrm{m}_{\mathrm{A}}=200 \mathrm{GeV} / \mathrm{c}^{2}, \tan \beta \sim 25$. As the mass of $\mathrm{Z}$ is lower than that of the Higgs boson, the leptons and jets have lower $\mathrm{p}_{\mathrm{T}}$, and the selection efficiency for the associated $\mathrm{Z} / \gamma^{*} \rightarrow \tau \tau$ events is lower. Therefore less gg/qq $\rightarrow \mathrm{b} \overline{\mathrm{bZ}} / \gamma^{*}, \mathrm{Z} / \gamma^{*} \rightarrow \tau \tau$ events are expected to be reconstructed than Higgs bosons decaying into $\tau \tau$. However, it is possible to study the mass reconstruction using inclusive $\mathrm{Z} / \gamma^{*} \rightarrow \tau \tau$ events with $\mathrm{e} \mu$ final states.

Other potential backgrounds are $\mathrm{b} \overline{\mathrm{b}}, \mathrm{WW}, \mathrm{WZ}$ and $\mathrm{ZZ}$ events. The contribution from these backgrounds turns out, however, to be negligible after event selection [8]. The cross sections for signal and main background processes are shown in Table 1. 


\begin{tabular}{|c|c|c|c|c|c|}
\hline Signal bbZ $/ \gamma^{*}$ & $\mathrm{GeV} / c^{2}$ & $\mathrm{pb}$ & Background & $\mathrm{GeV} / c^{2}$ & $\mathrm{pb}$ \\
\hline$\tau \tau \mathrm{bb}(\tau \rightarrow e / \mu)$ & $\left(60<\mathrm{m}_{\tau \tau}<100\right)$ & 3.29 & $\mathrm{Z} / \gamma^{*} \rightarrow \tau \tau(\tau \rightarrow e / \mu)$ & $\left(80<\mathrm{m}_{\tau \tau}<100\right)$ & 223.2 \\
\hline$\tau \tau \mathrm{b} \overline{\mathrm{b}}(\tau \rightarrow e / \mu)$ & $\left(\mathrm{m}_{\tau \tau}>100\right)$ & 0.132 & $\mathrm{Z} / \gamma^{*} \rightarrow \tau \tau(\tau \rightarrow e / \mu)$ & $\left(\mathrm{m}_{\tau \tau}>100\right)$ & 10.1 \\
\hline$\mu \mu \mathrm{b} \overline{\mathrm{b}}$ & $\left(60<\mathrm{m}_{\mu \mu}<100\right)$ & 26.2 & $\mathrm{Z} / \gamma^{*} \rightarrow \mu \mu$ & $\left(\mathrm{m}_{\mu \mu}>80\right)$ & 1891 \\
\hline$\mu \mu \mathrm{b} \overline{\mathrm{b}}$ & $\left(\mathrm{m}_{\mu \mu}>100\right)$ & 1.05 & $\mathrm{Z} / \gamma^{*} \rightarrow \mathrm{ee}$ & $\left(\mathrm{m}_{\mathrm{ee}}>80\right)$ & 1891 \\
\hline eeb $\bar{b}$ & $\left(60<\mathrm{m}_{\mathrm{ee}}<100\right)$ & 26.3 & $\mathrm{t} \overline{\mathrm{t}} \rightarrow \ell \ell$ & & 86.2 \\
\hline eeb $\bar{b}$ & $\left(m_{e e}>100\right)$ & 1.05 & $\mathrm{tW} \rightarrow \ell \ell$ & & 6.16 \\
\hline
\end{tabular}

Table 1: Cross sections for the signal and background processes.

\section{DETECTOR SIMULATION}

The detector simulation was done using full GEANT[9] simulation. The CMS detector was simulated with complete ideal detector, no staging and no misalignment of the detector elements was considered. The pile-up is also included in the simulation: for a luminosity of $2 \times 10^{33} \mathrm{~cm}^{-2} \mathrm{~s}^{-1}$ it consists of 3.4 minimum bias events superimposed per bunch crossing.

The standard CMS reconstruction code, ORCA [10], was used in this analysis. Muon and electron reconstruction efficiencies are respectively $95 \%$ and $85 \%$. For electrons, however, this efficiency drops to $52 \%$ when electron identification cuts [11] are applied. The ECAL+HCAL tower $\mathrm{E}$ and $\mathrm{E}_{\mathrm{T}}$ thresholds are, respectively, 0.8 and 0.5. Jets are reconstructed with an iterative cone algorithm, with cone size 0.5 , and energy scale corrections are applied as described in [12].

\section{EVENT SELECTION}

\subsection{Trigger}

The events were triggered with single and double electron and muon trigger. The $\mathrm{p}_{\mathrm{T}}$ threshold for single muons is $19 \mathrm{GeV} / \mathrm{c}$, for single electrons $26 \mathrm{GeV} / \mathrm{c}$, for double muons $7 \mathrm{GeV} / \mathrm{c}$ and for double electrons $14.5 \mathrm{GeV} / \mathrm{c}$ [13]. The Level 1 trigger efficiency for the signal was 0.914 . The overall trigger efficiency for signal, DY, $t \bar{t}$ and $t W$ backgrounds was found to be $0.826,0.895,0.821$ and 0.684 , respectively. Stronger trigger thresholds and lower efficiency on single and double electrons suppress the electron final states with respect to the muon final states. Therefore there are more muon events than electron events in the signal and background passing the trigger.

\subsection{Offline selection}

In this study there are three different goals in the event selection. The first goal is to measure the $\mathrm{Z}$ boson and $\mathrm{b}$ jet $\mathrm{p}_{\mathrm{T}}$ distributions to verify the kinematics. It is important to have as pure sample of $\mathrm{b} \overline{\mathrm{b} Z}$ events as possible, and strong cuts are needed. The second goal is to measure the $\mathrm{b} \overline{\mathrm{b}} \mathrm{Z}$ cross section. For the cross section measurement

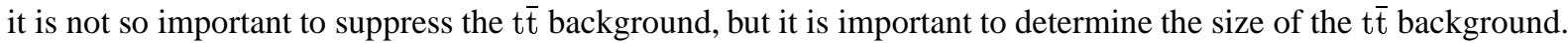
The third goal is to test the mass reconstruction method developed for $\mathrm{H} / \mathrm{A} \rightarrow \tau \tau$ events. For mass reconstruction it is important to have $\tau \tau$ events, so an electron-muon final state is chosen with loose cuts on jets, as extracting the $\mathrm{b} \overline{\mathrm{bZ}}$ events from inclusive Drell-Yan is not important. Therefore different sets of cuts are used for different goals.

The basic event selection is a requirement of two isolated oppositely charged leptons (e or $\mu$ ) with $\mathrm{p}_{\mathrm{T}}>20 \mathrm{GeV} / \mathrm{c}$ in the central detector acceptance region $|\eta|<2.5$ and coming from a reconstructed primary vertex. The leptons are defined isolated when there are no other tracks from the primary vertex with $\mathrm{p}_{\mathrm{T}}>1 \mathrm{GeV} / \mathrm{c}$ within a cone $\Delta \mathrm{R}=\sqrt{\Delta \varphi^{2}+\Delta \eta^{2}} \leq 0.4$ around the lepton. The $\mathrm{p}_{\mathrm{T}}$ cut and isolation reduce efficiently the backgrounds with soft leptons due to semileptonic decays of $\mathrm{b}$ and $\mathrm{c}$ quarks. Other methods which are used to suppress the backgrounds are $b$ tagging and central jet veto. The missing transverse energy (MET) is used to suppress t $\bar{t}$ events. Missing $\mathrm{E}_{\mathrm{T}}$ is also needed in the Higgs $\rightarrow \tau \tau$ mass reconstruction method due to neutrinos in the final state. Impact parameter, the minimum distance between the track trajectory and the primary interaction point, can be used to suppress events where leptons do not originate from tau decays, as taus travel a short distance $(c \tau \sim 87 \mu m)$ before they decay.

$\mathrm{B}$ jets associated with the $\mathrm{Z}$ boson provide a powerful tool to separate the $\mathrm{b} \overline{\mathrm{b}} \mathrm{Z} / \gamma^{*}$ events from the $\mathrm{Z} / \gamma^{*}$ background. The $\mathrm{Z} / \gamma^{*}$ events are mostly produced with no significant jet activity, and the associated jets are mostly light quark and gluon jets. Therefore the $\mathrm{Z} / \gamma^{*}$ background can be suppressed by requiring reconstructed jets present in the event, and even further by requiring that the associated jets are identified as $b$ jets. There are two possibilities available, either to require one $b$ tagged jet in the event and veto other jets, or to require two $b$ tagged jets in the 
event. Here the 2b-tagging option is used when more pure sample of $\mathrm{b} \overline{b Z} / \gamma^{*}$ events is needed. An $E_{T}$ threshold of $20 \mathrm{GeV}$ is used for both jets.

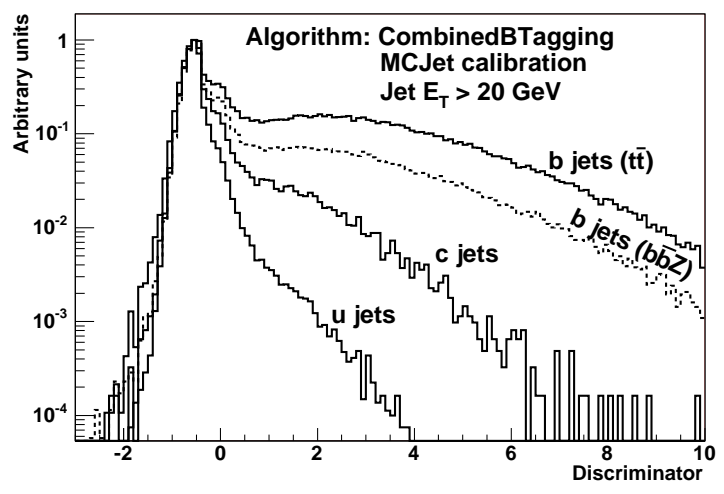

Figure 2: The output of $b$ tagging algorithm based on secondary vertices for $\mathrm{b}, \mathrm{c}$ and light quark and gluon jets in $\mathrm{t} \overline{\mathrm{t}}$ events.

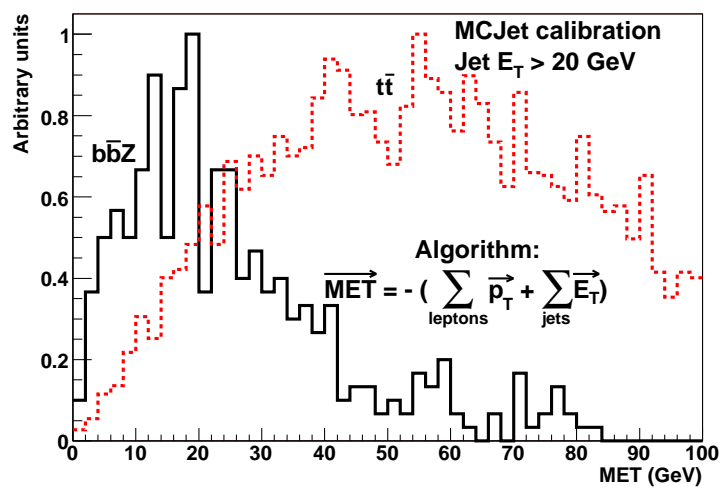

Figure 3: Missing $\mathrm{E}_{\mathrm{T}}$ (MET) distributions for $\mathrm{b} \overline{\mathrm{b} Z} / \gamma^{*}$ and $\mathrm{t} \overline{\mathrm{t}}$ events. MET is calculated from leptons and jets coming from the primary vertex.

$\mathrm{B}$ jets associated with the Higgs and $\mathrm{Z}$ bosons are generally very soft, which makes their tagging a challenging task. In a low energy jet the track multiplicity and momenta tend to be low, and many jets do not have enough significant tracks to be identified as $a b$ jet. As a consequence the $b$ tagging efficiency is not very high. In this study $a b$ tagging algorithm based on the reconstruction of the secondary decay vertex of the decaying $B$ hadron[14] is chosen. The b-Likelyhood (discriminator) of that algorithm is shown in Fig.2 for b, c and light quark and gluon jets in $t \bar{t}$ and $b$ jets in $\mathrm{b} \overline{b Z} / \gamma^{*}$ events. A cut of discriminator $>2$ gives on average $22 \% \mathrm{~b}$ tagging efficiency per jet $\left(\mathrm{b} \overline{\mathrm{bZ}} / \gamma^{*}\right)$ with $0.091 \%$ mistagging rate $\left(\mathrm{jjZ} / \gamma^{*}\right)$. A cut stronger than this suppresses the signal too much with

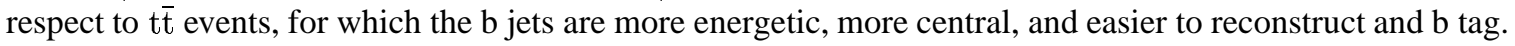

The $t \bar{t}$ events have more jet activity than $\mathrm{b} \overline{\mathrm{b} Z} / \gamma^{*}$, and a jet veto is used to suppress the $t \overline{\mathrm{t}}$ background. Events with jets $\mathrm{E}_{\mathrm{T}}>20 \mathrm{GeV}$ within the tracker acceptance region in addition to the two $\mathrm{b}$ jets are rejected. In mass reconstruction, where only one reconstructed jet is required, the events with more than one jet within the tracker acceptance region are rejected.

In $\mathrm{t} \overline{\mathrm{t}}$ events the leptons come from $\mathrm{W}$ decays, so there are always neutrinos in the final state. For the signal there are no neutrinos in the final state $\left(\mathrm{b} \overline{\mathrm{bZ}} / \gamma^{*} \rightarrow \mathrm{b} \overline{\mathrm{b}} \tau \tau\right.$ events with leptonic tau decays represent only about $6 \%$ of the signal cross section, and they are suppressed by lepton $\mathrm{p}_{\mathrm{T}}$ cut), and missing transverse energy is expected only due to measurement error. The missing $\mathrm{E}_{\mathrm{T}}$ is reconstructed from the high $\mathrm{E}_{\mathrm{T}}$ objects in the event: the two leptons, and the jets coming from the primary vertex. The lepton and jet origin is compared with the position of the primary vertex along the beam axis. A jet is defined to be coming from the primary vertex, if at least half of its tracks are coming from the primary vertex. A MCJet correction on the jet energy scale was used. The missing $\mathrm{E}_{\mathrm{T}}$ distributions for $\mathrm{b} \bar{b} Z / \gamma^{*}$ and $t \bar{t}$ events are shown in Fig. 3.

A strong method to separate the $\mathrm{Z}$ boson events from the $\mathrm{t} \overline{\mathrm{t}}$ background is to reconstruct the invariant $\mathrm{Z}$ mass. The two leptons are measured with a good accuracy, and the invariant mass distribution shows a clear peak at the $Z$ boson mass. The $t \bar{t}$ background is strongly suppressed when only events within $85<\mathrm{m}_{\ell \ell}<95 \mathrm{GeV} / \mathrm{c}^{2}$ are chosen.

A summary of the used cuts is shown in Table 2.

\section{RESULTS}

\subsection{Verification of Monte Carlo}

The verification of the Monte Carlo for $\mathrm{b} \overline{\mathrm{b}} \mathrm{Z} / \gamma^{*}$ events includes the verification of the $\mathrm{Z} \mathrm{p}_{\mathrm{T}}$ distribution, the associated $\mathrm{b}$ jet $\mathrm{E}_{\mathrm{T}}$ and $\eta$ distributions, and the cross section measurement. These has been studied in Ref. [15]. Each of these distributions consists of both signal and background events, the measured distribution is a sum of signal and different background distributions. The shapes of the $\mathrm{p}_{\mathrm{T}}, \mathrm{E}_{\mathrm{T}}$ and $\eta$ distributions for the backgrounds can be measured from data. The distributions for $t \bar{t}$ events can be measured from outside the signal region of the measured invariant mass, and the distributions for $\mathrm{jj} Z / \gamma^{*}$ events with relaxed $\mathrm{b}$ tagging. The contribution of 


\begin{tabular}{|l|c|c|c|}
\hline Cut & kinematics & cross section & mass reco \\
\hline Final state & ee $/ \mu \mu$ & ee $/ \mu \mu$ & $\mathrm{e} \mu$ \\
Lepton $\mathrm{p}_{\mathrm{T}}$, isolation & $\mathrm{p}_{\mathrm{T}}>20 \mathrm{GeV} / \mathrm{c}$ & $\mathrm{p}_{\mathrm{T}}>20 \mathrm{GeV} / \mathrm{c}$ & $\mathrm{p}_{\mathrm{T}}>20 \mathrm{GeV} / \mathrm{c}$ \\
$\mathrm{Q}\left(\ell_{1}\right) \times \mathrm{Q}\left(\ell_{2}\right)=-1$ & yes & yes & yes \\
$\sigma_{\text {ip }}\left(\ell_{1}\right) \oplus \sigma_{\mathrm{ip}}\left(\ell_{2}\right)$ & no & no & $>5$ \\
$\mathrm{~b}$ tagging & $2 \mathrm{~b} \mathrm{tag,} \mathrm{discr}>2$ & $2 \mathrm{~b} \mathrm{tag,} \mathrm{discr}>2$ & no b tagging \\
jet veto & $\mathrm{N}_{\text {jets }}=2$ & $\mathrm{~N}_{\text {jets }}=2$ & $\mathrm{~N}_{\text {jets }}=1$ \\
MET & $\mathrm{MET}<30 \mathrm{GeV}$ & $\mathrm{MET}<50 \mathrm{GeV}$ & no \\
$85<\mathrm{m}_{\ell \ell}<95 \mathrm{GeV} / c^{2}$ & yes & no & no \\
$\Delta \varphi\left(\ell_{1}, \ell_{2}\right)<175^{\circ}$ & no & no & yes \\
$\mathrm{E}_{\nu}\left(\tau_{1}\right)>0, \mathrm{E}_{\nu}\left(\tau_{2}\right)>0$ & no & no & yes \\
\hline
\end{tabular}

Table 2: Sets of cuts used for different measurements.

$\mathrm{t} \overline{\mathrm{t}}$ events can be measured from data by integrating the background fit on the invariant mass distribution over the chosen $\mathrm{Z}$ boson mass window $85-95 \mathrm{GeV} / \mathrm{c}^{2}$. The $\mathrm{jjZ} / \gamma^{*}$ contribution can be estimated using the known $\mathrm{b}$ tagging efficiency and mistagging rate. These efficiencies can be measured from data using t $\bar{t}$ events [16]. When no $b$ tagging is used, the fraction of $\mathrm{Z} / \gamma^{*}$ events associated with two genuine $\mathrm{b}$ quarks is expected to be of the order of $1 \%$. The number of mistagged events after $2 \mathrm{~b}$-tagging is then $\mathrm{N}_{\mathrm{jjZ} / \gamma^{*}}=\epsilon_{\text {mistag }}\left(\mathrm{jet}_{1}\right) \times \epsilon_{\text {mistag }}\left(\mathrm{jet}_{2}\right) \times \mathrm{N}_{\mathrm{jjZ} / \gamma^{*}}^{\text {nobtag }}$. The fraction of events with associated $\mathrm{c}$ jets can be estimated by using the fraction of $\mathrm{b} \overline{\mathrm{b} Z} / \gamma^{*}$ and $c \overline{\mathrm{c} Z} / \gamma^{*}$ cross sections, provided by theory calculations [17], and the fraction of $\mathrm{b}$ tagging efficiency versus mistagging rate for $\mathrm{c}$ jets provided by Monte Carlo simulations [12]. The above fraction of the cross sections is about one (1.224) [17]. The shapes of the distributions together with the estimated fractions for signal and background contributions can be used to estimate the shape of the summed distributions, which can then be compared with the measured ones.

The $\mathrm{b}$ tagged jet $\mathrm{E}_{\mathrm{T}}$ and $\eta$ distributions are shown in Figs. 4 and 5. The solid histogram represent the measured distributions, the dashed and dotted histograms the contributions from different components. The event selection described in Table 2 for kinematics verification was used. From the total number of passed events (1065 events for $30 \mathrm{fb}^{-1}$ ) the fraction of signal events $\left(\mathrm{b} \overline{\mathrm{b} Z} / \gamma^{*}\right)$ is $70 \%$ (dashed histograms), the fraction of $\mathrm{Z} / \gamma^{*}$ with mistagged jets is $11 \%$, and the fraction of $\mathrm{t} \overline{\mathrm{t}}$ events is $19 \%$. All other backgrounds are negligible. The contribution from $\mathrm{Z}, \gamma^{*}$ events with soft initial and final state radiation mistagged as bjets comes mostly at low values of jet $\mathrm{E}_{\mathrm{T}}$. This is shown as a gap between the points and the solid histogram in the figures. Due to the hard $\mathrm{b}$ tagging cut used the purity of the measured $\mathrm{b}$ jets in $\mathrm{b} \overline{\mathrm{b} Z} / \gamma^{*}$ events is very high, close to one, and dropping to 0.96 at low values of jet $\mathrm{E}_{\mathrm{T}}$.

The $\mathrm{Z}$ boson $\mathrm{p}_{\mathrm{T}}$ reconstructed from the two leptons is shown in Fig. 6. Again the shapes of the background

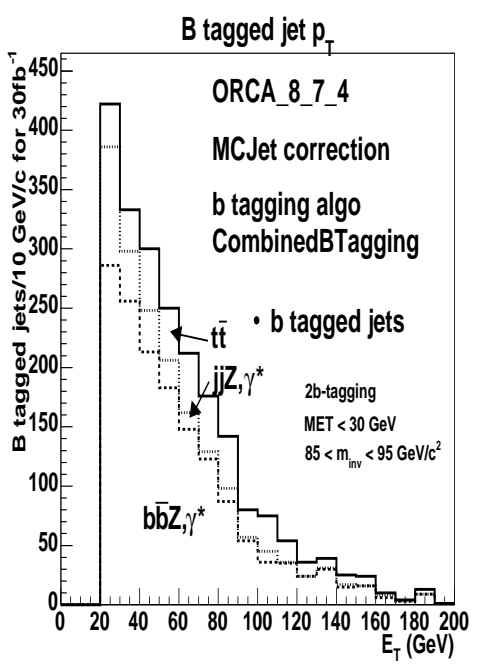

Figure 4: Reconstructed and $b$ tagged jet $\mathrm{p}_{\mathrm{T}}$ (solid) and the fraction of $\mathrm{b} \overline{\mathrm{b} Z} / \gamma^{*}$ (dashed) and $\mathrm{jjZ} / \gamma^{*}$ events (dotted histogram).

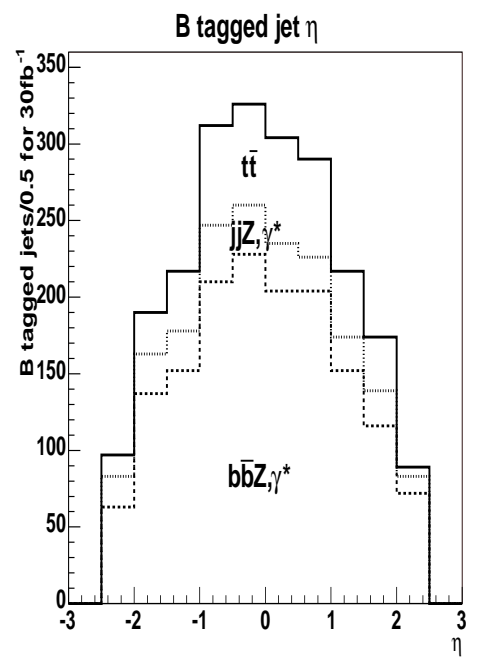

Figure 5: Reconstructed and b tagged jet $\eta$ (solid) and the fraction of $\mathrm{b} \overline{\mathrm{bZ}} / \gamma^{*}$ (dashed) and $\mathrm{jjZ} / \gamma^{*}$ events (dotted histogram).

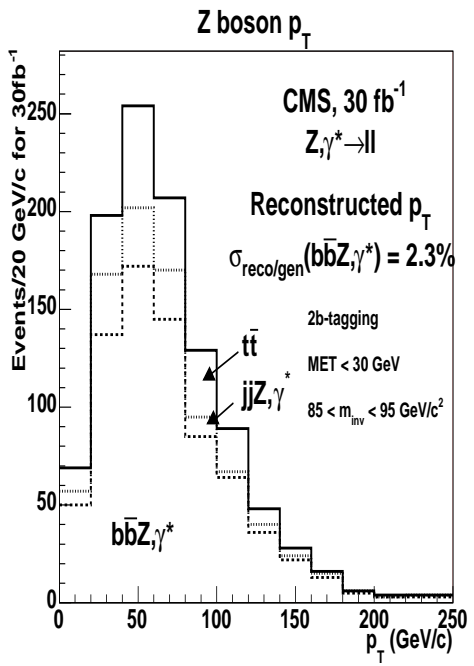

Figure 6: $\mathrm{Z}$ boson $\mathrm{p}_{\mathrm{T}}$ reconstructed from the two leptons (solid) and the fraction of $\mathrm{b} \overline{\mathrm{b} Z} / \gamma^{*}$ (dashed) and $\mathrm{jjZ} / \gamma^{*}$ events (dotted histogram). 
distributions and their contributions are needed. Since the $\mathrm{Z}$ boson $\mathrm{p}_{\mathrm{T}}$ is reconstructed from the two well measured leptons, the difference between the reconstructed and generated $\mathrm{Z}$ boson $\mathrm{p}_{\mathrm{T}}$ is small, about $2.3 \%$.

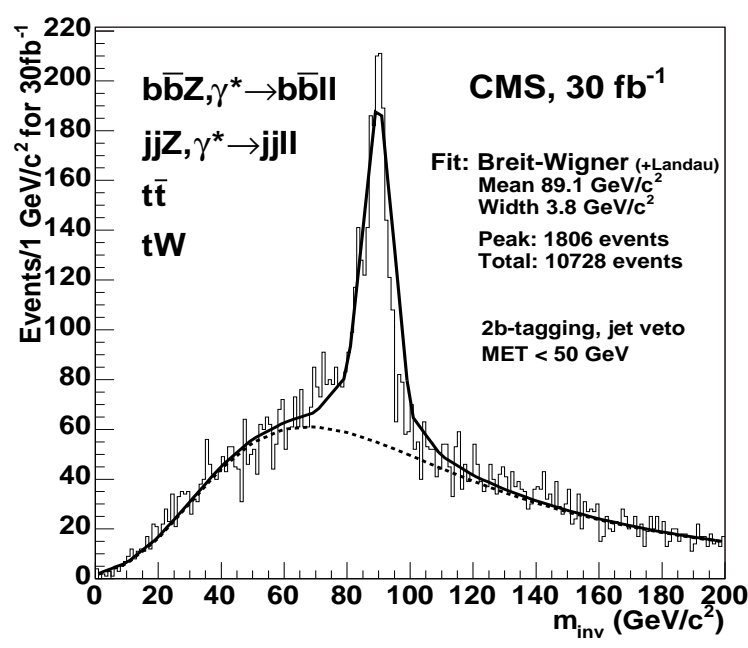

Figure 7: Invariant mass of the two leptons.

In the cross section measurement it is important to have a clear and easily measured Z boson peak over the background. This is achieved with the event selection shown in Table 2 for cross section measurement. The invariant mass $m_{\ell \ell}$ distribution is shown in Fig. 7. The number of background events consisting mostly on $t \bar{t}$ and $t W$ events can be estimated from the fit with good statistics. The number of measured $\mathrm{Z}$ boson events, here 1806 events for 30 $\mathrm{fb}^{-1}$, consists of $\mathrm{b}$ tagged $\mathrm{b} \overline{\mathrm{b} Z} / \gamma^{*}$ events and mistagged $\mathrm{jj} \mathrm{Z} / \gamma^{*}$ events with no genuine $\mathrm{b}$ quark content. The fraction of mistagged events can be estimated using the known b tagging efficiency and mistagging rate as described for the kinematics measurement. The difference between the measured events and the estimated mistagged events gives the number of signal events $\mathrm{N}_{\mathrm{b} \overline{b Z} / \gamma^{*}}$, which can be turned into a cross section.

\subsection{Systematic uncertainties}

The uncertainty on the signal selection efficiency is related to the uncertainty of the lepton identification, the absolute calorimeter scale and the b tagging efficiency. An error in calorimeter scale introduces an error in the jet energy measurement. Here a $2 \%$ error on calorimeter scale, simulated by varying the jet energies, leads to a $5.5 \%$ error on the signal selection efficiency. The uncertainty of the $b$ tagging efficiency can be estimated from $t \bar{t}$ events as in Ref. [16]. A value of 5\% can be used as a conservative estimate. A lepton identification uncertainty of $2 \%$ is used for both electrons and muons. The uncertainty of the two jets and two leptons are added linearly due correlations on errors, giving b tagging uncertainty of $10 \%$ for the two jets and lepton identification uncertainty of $4 \%$ for the two leptons.

The uncertainty of the $t \bar{t}$ background can be evaluated from the signal+background fit shown in Fig.7. The $t \bar{t}$ background is evaluated from the signal+background fit shown in Fig.7 The uncertainty of the number of jjZ/ $\gamma^{*}$ events $\mathrm{N}_{\mathrm{jjZ} / \gamma^{*}}=\epsilon_{\text {mistag }}\left(\right.$ jet $\left._{1}\right) \times \epsilon_{\text {mistag }}\left(\right.$ jet $\left._{2}\right) \times \mathrm{N}_{\mathrm{jjZ} / \gamma^{*}}^{\text {nobtag }}$ is $\Delta \mathrm{N}_{\mathrm{jjZ} / \gamma^{*}} / \mathrm{N}_{\mathrm{jjZ} / \gamma^{*}}=\sigma_{\text {fit }} \oplus 2 \sigma_{\text {mistag }}$, where $\sigma_{\mathrm{fit}}$ is the uncertainty of the $\mathrm{Z}$ peak fit when no $\mathrm{b}$ tagging is used. Assuming a $5 \%$ mistagging uncertainty and a $1.7 \%$ error from the $\mathrm{Z}$ peak fit without b tagging, the uncertainty of the $\mathrm{jjZ} / \gamma^{*}$ background is $\Delta \mathrm{N}_{\mathrm{jjZ} / \gamma^{*}} / \mathrm{N}_{\mathrm{jjZ} / \gamma^{*}}=10.1 \%$.

The total systematic uncertainty of the above measurements including the luminosity uncertainty of 5\%[12] yields a $16.6 \%$ uncertainty on the cross section measurement. The variation of the luminosity and it's effect on pile-up is not taken into account.

\subsection{Mass reconstruction}

In $\mathrm{H}_{\mathrm{SUSY}} \rightarrow \tau \tau$ analysis the Higgs boson mass is reconstructed using a collinear approximation method. Due to neutrinos in the final state a precise mass reconstruction is impossible. In collinear approximation the neutrinos are assumed to be emitted along the leptons, which is a valid assumption for the signal events due to large Lorentz boost of the two $\tau$ 's. The missing transverse energy is projected along the lepton transverse momentum directions, giving 
an estimate for the neutrino momentum including the $\mathrm{z}$ component of the neutrino momentum. The reconstructed mass is the invariant mass of the summed lepton and neutrino 4-momenta.

Mass reconstruction using the collinear approximation is possible when the two leptons are not in a back-to-back configuration in the transverse plane. Events in back-to-back configuration are removed with a cut $\Delta \varphi\left(\ell_{1}, \ell_{2}\right)<$ $175^{\circ}$, where $\Delta \varphi\left(\ell_{1}, \ell_{2}\right)$ is the angle between the two leptons in the transverse plane. The mass reconstructed using the collinear approximation is shown in Fig.8 using event selection listed in Table 2 for mass reconstruction. The $\mathrm{e}+\mu$ final states were chosen to select events with intermediate $\tau$ 's. In the Higgs boson studies one b jet is required present in the event with a veto on additional jets. Similar events were chosen here, one associated jet was required in the event, but to have better statistics no b tagging was used. Since the leptons are generally well measured, the mass peak position and width are highly dependent on the quality of the missing transverse energy measurement. Here the mean value of the mass $99 \mathrm{GeV} / c^{2}$ is within one sigma error of the correct value. This measured value can be used to verify also the detector calibration, a better calibration should result in a measured mass closer to the known $\mathrm{Z}$ boson mass.

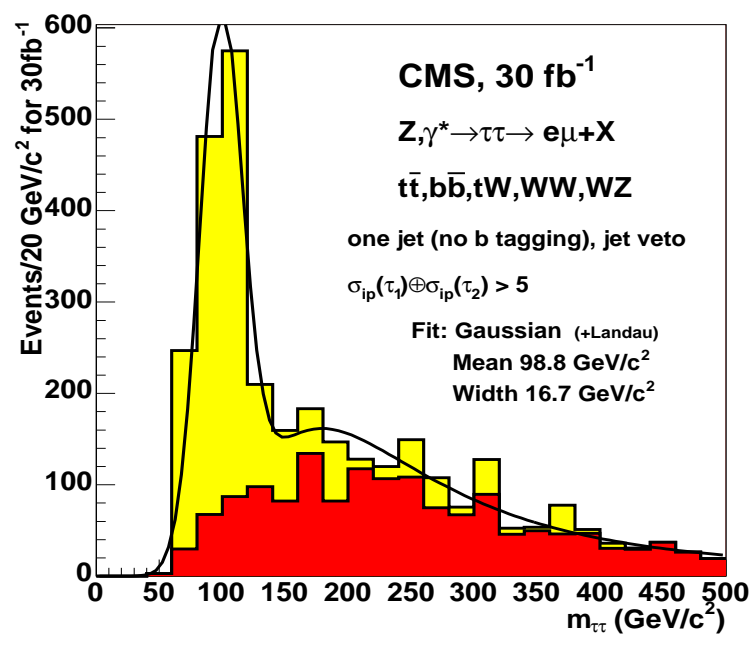

Figure 8: Mass reconstructed using collinear approximation.

\section{CONCLUSIONS}

It is shown that the $\mathrm{b} \overline{\mathrm{bZ}} / \gamma^{*}$ events can be used as a benchmark for the MSSM Higgs production gg/qq $\rightarrow \mathrm{b} \overline{\mathrm{b}} \mathrm{H}$. $\mathrm{The} \mathrm{b} \overline{\mathrm{bZ}} / \gamma^{*}$ cross section can be measured and compared with the highest order theoretical calculation available. The uncertainty of the cross section measurement is expected to be about $17 \%$. The associated jet $\mathrm{E}_{\mathrm{T}}$ and $\eta$ distributions as well as $\mathrm{Z}_{\mathrm{T}}$ distribution can be measured and compared with the expected theoretical distributions. The uncertainty of the $\mathrm{Z}$ boson $\mathrm{p}_{\mathrm{T}}$ measurement is $2.3 \%$ due to well measured lepton momenta. The Higgs boson reconstruction methods can be tested with $\mathrm{Z}$ bosons from real data. Understanding the $\mathrm{b} \overline{\mathrm{b}} \mathrm{Z} / \gamma^{*}$ events helps us to understand and better trust the theoretical predictions for $\mathrm{b} \overline{\mathrm{b}} \mathrm{H}$ events, if a heavy neutral MSSM Higgs boson is found in a $\mathrm{H} \rightarrow \tau \tau$ final state.

\section{ACKNOWLEDGEMENTS}

The author would like to thank the ARDA/ASAP team for a magnificent grid tool they have created, and V. Ciulli, P. Garcia, R. Kinnunen, S. Lowette and A. Nikitenko for reading the manuscript.

\section{References}

[1] F. Maltoni, T. McElmurry, and S. Willenbrock, Phys. Rev. D72 (2005) 074024, [hep-ph/ 0505014$].$

[2] S. Abdullin, S. Banerjee, L. Bellucci, C. Charlot, D. Denegri, M. Dittmar, V. Drollinger, M. Dubinin, M. Dzelalija, D. Green, I. Iashvili, V. Ilyin, R. Kinnunen, S. Kunori, K. Lassila-Perini, S. Lehti, 
K. Mazumdar, F. Moortgat, T. Muller, A. Nikitenko, I. Puljak, P. Salmi, C. Seez, S. Slabospitsky, S. Stepanov, R. Vidal, W. Wu, H. Yildez, and M. Zeyrek, CMS Note 2003/006.

[3] A. Pukhov, E. Boos, M. Dubinin, V. Edneral, V. Ilyin, D. Kovalenko, A. Kryukov, V. Savrin, S. Shichanin, and A. Semenov, INP-MSU 98-41/542 [hep-ph/9908288].

[4] T. Sjostrand, L. Lonnblad, S. Mrenna, and P. Skands, LU TP 03-38 (2003) [hep-ph/ 0308153 ].

[5] S. Slabospitsky and L. Sonnenschein, Comput. Phys. Commun. 148 (2002) 87, [hep-ph/0201292].

[6] J. Campbell, hep-ph/0105226.

[7] CERN Yellow Report (2000) [hep-ph/0003033].

[8] S. Lehti, CMS Note 2002/035.

[9] GEANT4 Collaboration, S.Agostinelli et. al., Nucl. Instrum. and Methods A506 (2003) 250-303.

[10] ORCA: CMS Reconstruction Package. Site located at http://cmsdoc.cern.ch/orca.

[11] G. Davattz, M. Dittmar, and A.-S. Giolo-Nicollerat, CMS Note 2006/047.

[12] CERN/LHCC 2006-001 CMS TDR 8.1 (2006).

[13] CERN/LHCC 02-26 CMS TDR 6 (2002).

[14] C. Weiser, CMS Note 2006/014.

[15] J. Campbell, R. K. Ellis, and D. L. Rainwater, Phys. Rev. D68 (2003) 094021, [hep-ph / 0308195 ].

[16] S. Lowette, J. D’Hondt, and J. Heyninck, CMS Note 2006/013.

[17] J. Campbell, R. K. Ellis, F. Maltoni, and S. Willenbrock, hep-ph/0510362. 